\title{
Blickdiagnose
}



\section{Vulvakarzinom auffällig pigmentiert}

Eine 62-jährige Patientin stellt sich mit einem nicht heilenden Geschwür am äußeren Genitale vor. Es findet sich ein exulzerierender Tumor im Bereich der Klitoris und der vorderen Vulva. Die Urethralöffnung ist noch frei.

In der Regel handelt es sich bei solchen Tumoren um Plattenepithelkarzinome der Vulva. Allerdings sieht man im Randbereich des Tumors rechts und auf der Schleimhaut rechts kaudal eine auffällige Hyperpigmentierung. Die Gewebeuntersuchung zeigt ein Melanom.
Vulvamelanome kommen häufiger vor, als es aufgrund der Körperoberfläche der Vulva zu erwarten wäre. Der Altersgipfel liegt in der fünften und sechsten Lebensdekade. Die Therapie besteht in einer radikalen Resektion mit Entfernung inguinaler und eventuell pelviner Lymphknoten. Die Prognose ist ungünstig mit einer Fünfjahresüberlebensrate von nur $30-35 \%$.

- OA Dr. med. Olaf Buchweitz, Univ.-Prof. Dr. med. Ralph J. Lellé, MIAC, Universitäts-Frauenklinik Münster, Albert-SchweitzerStraße 33, D-48149 Münster

\section{Ihr besonderer Fall?}

Sicher sehen auch Sie $a b$ und an einen besonders eindrucksvollen Befund in Ihrer Praxis. Fotografieren Sie ihn, schreiben Sie uns unter dem Stichwort Blickdiagnose, bei Veröffentlichung erhalten Sie 100 Euro.

MMW-Fortschritte der Medizin

E-Mail: manhart@urban-vogel.de

Fax: 089/4372-1420

Weitere interessante Blickdiagnosen finden Sie in unserem Online-Archiv unter www.mmw.de. 